\title{
Checklist dos Conopidae (Insecta, Diptera) do Estado do Mato Grosso do Sul, Brasil
}

\author{
Leonardo Silvestre Gomes Rocha \& Cátia Antunes de Mello-Patiu²
}

1. Instituto Federal de Educação, Ciência e Tecnologia do Rio de Janeiro, Campus Nilópolis, RJ, Brasil.
2. Departamento de Entomologia, Museu Nacional/UFRJ, Quinta da Boa Vista, São Cristóvão, 20940-040 Rio de Janeiro, RJ, Brasil. (camello@acd.ufrj.br)

Recebido 29 novembro 2016

Aceito 6 fevereiro 2017

DOI: $10.1590 / 1678-4766 e 2017144$

\begin{abstract}
Checklist of Conopidae (Insecta, Diptera) from Mato Grosso do Sul State, Brazil. Conopidae is a poorly studied family and its systematics is controversial among the Diptera. The adults are nectarivorous and the larvae are parasitic in Hymenoptera, Orthoptera, Blattaria and Diptera. Eight hundred species in 56 genera are known, with worldwide distribution. A list of 15 species in four genera recorded from Mato Grosso do Sul State, based on the available bibliography and the identified material from several Brazilian collections, is provided. The collections are concentrated in Maracaju municipality, in Cerrado biome, with only one exception. It is necessary to increase the sampling in other regions and environments of the state, to fill the sampling gaps.
\end{abstract}

KEYWORDS. Fauna, biodiversity, Pantanal, Cerrado, Biota-MS Program.

RESUMO. Conopidae é uma família pouco estudada e de sistemática controversa entre os Diptera. Os adultos são nectarívoros e as larvas têm hábito parasitóide em Hymenoptera, Orthoptera, Blattaria e Diptera. São conhecidas 800 espécies e 56 gêneros com distribuição mundial. Uma lista de 15 espécies em quatro gêneros registradas para o estado do Mato Grosso do Sul, elaborada com base na bibliografia disponível e no material identificado de diversas coleções brasileiras, é fornecida. As coletas estão concentradas no município de Maracaju em bioma de Cerrado, com apenas uma exceção. Faz-se necessário incrementar as coletas em outras regiões e outros ambientes do estado, para que lacunas de amostragem sejam preenchidas.

PALAVRAS-CHAVE. Fauna, biodiversidade, Pantanal, Cerrado, Programa Biota-MS.

Conopidae é uma família de dípteros com distribuição mundial, com cerca de 800 espécies em 56 gêneros (PAPE \& Thompson, 2010). Cinco subfamílias são propostas por GiBson et al. (2010): Conopinae, Myopinae, Dalmanniinae, Zodioninae e Stylogastrinae. A sistemática é controversa, com a família sendo considerada tradicionalmente como a única componente da superfamília Conopoidea, com posição basal entre os Schizophora e grupo-irmão de Tephritoidea (McAlpine, 1989). Outros trabalhos, porém, contestam essa classificação, tanto do ponto de vista das análises morfológicas quanto das moleculares, como em KoRNEYEv (1999) e HaN \& Ro (2004), que propuseram Conopidae como um táxon basal de Schizophora, porém grupo-irmão de Diopsidae. Dentre as propostas mais recentes de classificação, a filogenia baseada na análise molecular de GiBson et al. (2010), dependendo do método de análise, ou corrobora a filogenia morfológica ou sugere o grupo (Conopidae + Lauxanioidea) como irmão de todos os Schizophora.

Os adultos são alongados e esguios, e mimetizam himenópteros. Têm probóscide bem desenvolvida, adaptada ao hábito alimentar nectarívoro, alimentando-se em flores de plantas das famílias Apiaceae, Asteraceae e Lamiaceae (Lopes, 1937; Smith \& Peterson, 1987). As larvas são endoparasitas de outros insetos, preferencialmente os da ordem Hymenoptera, sendo consideradas pragas menores em apicultura (Smith, 1966; SMIth \& VAn Someren, 1970; Smith \& Peterson, 1987), exceto para os Stylogastrinae, que ovipositam em Orthoptera, Blattaria e Diptera Calyptratae (Lopes, 1937; SMith, 1967, 1969; Couri \& Pont, 2006). Têm importância menor como polinizadores, mas no geral a biologia é pouco conhecida, com poucos trabalhos concentrados nas espécies que parasitam himenópteros.

O conhecimento da fauna desta família tem sido seriamente afetado pelas numerosas lacunas de conhecimento, principalmente pelo baixo esforço de coleta, em especial na Região Neotropical. Assim, inventários faunísticos são ferramentas importantes para se evidenciar tais lacunas e apontar áreas onde podem haver novos dados de distribuição ou até mesmo a ocorrência de novas espécies, contribuindo para o aumento do conhecimento sobre este pouco estudado grupo.

\section{MATERIAL E MÉTODOS}

A listagem foi baseada no catálogo de PAPAVERo (1971) e nas informações retiradas do Biosystematic Database 
of World Diptera - BDWD (PAPE \& ThOMPSon, 2010). Para a subfamília Conopinae, adicionaram-se registros obtidos da literatura, como as revisões de CAMras $(1955,1957)$, e material identificado da Coleção Entomológica do Museu Nacional/UFRJ, Rio de Janeiro (MNRJ); para Stylogastrinae, complementou-se as informações com aquelas obtidas em CAMras \& PARrilllo (1985), e por material identificado das coleções do MNRJ, do Museu de Zoologia da USP, São Paulo (MZUSP), do Departamento de Zoologia, Universidade Federal do Paraná, Curitiba (DZUP), do Instituto Nacional de Pesquisas da Amazônia, Manaus (INPA) e do Museu Paraense Emílio Goeldi, Belém (MPEG).

Para cada espécie foram citadas a localidade-tipo e a distribuição geográfica conhecida. $\mathrm{Na}$ distribuição geográfica foram listados os países em sentido norte-sul e, para o Brasil, foram incluídos os estados usando-se as siglas oficiais apresentadas pelo IBGE (2012). Nos registros referentes ao Mato Grosso do Sul (MS) foram citados os municípios de ocorrência, quando possível.

\section{RESULTADOS E DISCUSSÃO}

\section{Lista dos Conopidae do Estado do Mato Grosso do Sul, Brasil}

\section{CONOPINAE}

Physocephala aurifrons (Walker, 1849). Localidade-tipo: desconhecida. Distribuição: México, Trinidad, Peru, Brasil [MS (Maracaju) a BA], Paraguai.

Physocephala bicolor Kröber, 1915. Localidade-tipo: Brasil, Mato Grosso do Sul, Maracaju. Distribuição: Brasil [MS (Maracaju)].

Physocephala unicolor Kröber, 1915. Localidade-tipo: Paraguai, La Cordillera, San Bernardino. Distribuição: Brasil [MS (Maracaju)], Paraguai, Argentina.

Physoconops abruptus Kröber, 1915. Localidadetipo: Argentina, Mendoza. Distribuição: Brasil [MS (Maracaju), SC], Paraguai, Argentina.

Physoconops apicalis Camras, 1955. Localidade-tipo: Brasil, Mato Grosso do Sul, Maracaju. Distribuição: Peru, Brasil [MS (Maracaju), SC].

Physoconops gilmorei Camras, 1955. Localidade-tipo: Brasil, Mato Grosso do Sul, Maracaju. Distribuição: Brasil [MS (Maracaju), GO], Paraguai.

Physoconops guianicus Curran, 1934. Localidade-tipo: Guiana, Bartica, Kartabo. Distribuição: Colômbia, Guiana, Venezuela, Peru, Brasil [MS (Maracaju), GO]. Physoconops infuscatus Camras, 1955. Localidade-tipo: Brasil, Mato Grosso do Sul, Maracaju. Distribuição: Brasil [MS (Maracaju) a SP, SC].

Physoconops nitens Camras, 1955. Localidade-tipo: Brasil, Mato Grosso do Sul, Maracaju. Distribuição: Brasil [MS (Maracaju), SC].

Physoconops ornatifrons Krober, 1915. Localidadetipo: Peru. Distribuição: Venezuela, Peru, Brasil [MS (Maracaju) a SC].

Physoconops shannoni Camras, 1955. Localidade-tipo:
Brasil, Mato Grosso do Sul, Maracaju. Distribuição: Brasil [MS (Maracaju)].

Physoconops travassosi Camras, 1955. Localidade-tipo: Brasil, Mato Grosso do Sul, Maracaju. Distribuição: Brasil [MS (Maracaju), GO, RJ, SP].

Tropidomyia alexanderi Camras, 1955. Localidade-tipo: Brasil, Mato Grosso do Sul, Maracaju. Distribuição: Brasil [MS (Maracaju), GO, SP], Paraguai.

\section{STYLOGASTRINAE}

Stylogaster rectinervis Aldrich, 1930. Localidade-tipo: Guiana, Bartica, Kartabo. Distribuição: Costa Rica, Colômbia, Equador, Peru, Guiana, Brasil [AP, RR, AM, PA, RO, TO, MG, RJ, SP, MS (Maracaju), SC].

Stylogaster stylata (Fabricius, 1805). Localidade-tipo:

"America Meridionalis". Distribuição: Colômbia, Equador, Brasil [PA, RJ, MS (Porto Murtinho), SC, RS], Paraguai, Argentina.

Foram registradas 15 espécies em quatro gêneros para o Estado do Mato Grosso do Sul. O município de Maracaju foi o único município com ocorrências registradas, com apenas uma exceção: Stylogaster stylata em Porto Murtinho. Adicionalmente, Maracaju foi identificada como a localidadetipo de oito das 14 espécies registradas no município. $\mathrm{O}$ Mato Grosso do Sul é atípico em relação ao restante do país, por apresentar uma maior riqueza de espécies para Physoconops Szilády, enquanto o gênero dominante no Brasil é Stylogaster Macquart.

O estado apresenta uma grande extensão territorial e variedade de biomas, com regiões de Cerrado, Floresta Atlântica e Pantanal. A maioria dos registros de Conopidae está concentrada em uma região dominada pelo Cerrado, mas o Pantanal representa a única exceção, com S. stylata. A fauna de conopídeos no estado é composta, em sua maioria, por espécies que têm ampla distribuição geográfica. No caso de Stylogaster, cujas espécies ocorrem em ampla variedade de ambientes, as duas espécies registradas ocorrem comprovadamente em regiões de Floresta Atlântica, Floresta Amazônica, Cerrado e Pantanal.

O conhecimento da fauna de Conopidae sofre uma grande distorção devido aos esforços de coleta não contínuos, tanto no Brasil quando no exterior. No caso do Mato Grosso do Sul, como muitas outras áreas do Brasil, o conhecimento é extremamente fragmentário e novos pontos de coleta fazem-se necessários para um entendimento mais completo e contínuo da fauna em seus diferentes ambientes. Portanto, verifica-se que o conhecimento da distribuição geográfica da maioria das espécies neotropicais de Conopidae ainda é muito preliminar e, provavelmente, suas espécies são mais amplamente distribuídas do que se conhece atualmente. A análise prévia da distribuição de algumas espécies mais bem amostradas revela as características de ampla distribuição e variedade de habitats, demonstrando a necessidade de coletas mais abrangentes e contínuas em todos os biomas do Brasil.

Principais grupos de pesquisa e acervos. Conopidae é pouco estudada no mundo, sendo os principais grupos de 
pesquisa dispersos pelo globo. Na América Latina, o único grupo a trabalhar com a família é o do Museu Nacional/ UFRJ, coordenado pela Profa. Cátia Mello-Patiu, com foco principal na sistemática de Sarcophagidae e Conopidae. Há também pesquisadores na Alemanha, Austrália, Canadá e Estados Unidos. No Brasil, as principais coleções com material identificado são as do Departamento de Zoologia, UFPR (DZUP), Instituto Nacional de Pesquisas da Amazônia (INPA), Museu Nacional/UFRJ (MNRJ), Museu Paraense Emílio Goeldi (MPEG) e Museu de Zoologia, USP (MZUSP).

Principais lacunas de conhecimento e perspectivas para os próximos 10 anos. Com relação ao Mato Grosso do Sul, como as coletas foram até hoje massivamente concentradas em Maracaju, no Cerrado, podemos apontar que é necessário realizar amostragens em outras regiões do estado, preferencialmente nos outros biomas, como a Floresta Atlântica e o Pantanal, onde se estima encontrar um número muito maior de espécies do que os já registrados. O projeto em desenvolvimento Sisbiota Diptera $(\mathrm{CNPq} /$ Fapesp), coordenado pelo Prof. Carlos Lamas (MZUSP), que abrange coletas no Mato Grosso, Mato Grosso do Sul e Rondônia traz perspectivas promissoras para o conhecimento dos conopídeos do MS.

Com relação aos estudos dos conopídeos neotropicais, a principal lacuna também se apoia na necessidade de coletas em toda a região, para suprir a carência de conhecimento da fauna desta família, seguida dos desmembramentos deste conhecimento, como a taxonomia, a filogenia, os inventários, as chaves de identificação, etc. A escassez de especialistas trabalhando com o grupo, especialmente na América do Sul, também deve ser apontada como uma importante lacuna e, em decorrência deste fato, muitas coleções do Brasil e de outros países possuem valioso material ainda não identificado. O aumento do conhecimento da diversidade de conopídeos e sua representatividade em diferentes regiões também possibilitarão o desenvolvimento de estudos biogeográficos, com enfoque amplo ou de alguns gêneros, ainda inexistentes. Tais estudos poderão apontar as regiões e ambientes onde deve se concentrar maior esforço de coleta.

Atualmente, o Prof. Leonardo Silvestre G. Rocha, um dos autores deste checklist, é especialista nesta família. Desenvolveu seu projeto doutorado envolvendo filogenia e biogeografia de Stylogaster.

Agradecimentos. A Fundação de Apoio ao Desenvolvimento do Ensino, Ciências e Tecnologia do Estado de Mato Grosso do Sul (Fundect) e a Superintendência de Ciências e Tecnologia do Estado de Mato Grosso do Sul (Sucitec/MS) pelo convite de participação neste fascículo especial da Iheringia, Série Zoologia e o suporte financeiro para sua publicação.

\section{REFERÊNCIAS BIBLIOGRÁFICAS}

Camras, S. 1955. A review of the New World flies of the genus Conops and allies (Diptera: Conopidae). Proceedings of the United States National Museum 105:155-187.

Camras, S. 1957. A review of the New World Physocephala (Diptera: Conopidae). Annals of the Entomological Society of America 50:213-218.

Camras, S. \& Parrillo, P. P. 1985. Review of the New World Stylogaster (Diptera Conopidae). Annals of the Entomological Society of America 78:111-126.

Couri, M. S. \& PonT, A. 2006. Eggs of Stylogaster Macquart (Diptera, Conopidae) on Madagascan muscids (Diptera, Muscidae). Proceedings of the California Academy of Sciences 57:473-478.

Gibson, J. F.; Skevington, J. H. \& Kelso, S. 2010. Placement of Conopidae within Schizophora based in mtDNA and nrDNA gene regions. Molecular Phylogenetics and Evolution 56:91-103.

HAN, H. Y. \& Ro, K. E. 2004. Molecular phylogeny of the superfamily Tephritoidea (Insecta: Diptera): New evidence from the mitochondrial $12 \mathrm{~S}, 16 \mathrm{~S}$ and COII genes. Molecular Phylogenetics and Evolution 34:416-430.

IBGE. Instituto Brasileiro de Geografia e Estatística. 2012. Disponível em: $<$ http://www.ibge.gov.br/estadosat/perfil.php?sigla=ms\#> Acessado em 30.08.2012.

Korneyev, V. A. 1999. Phylogenetic Relationships among the families of the Superfamily Tephritoidea. In: Aluja, M. \& Norrbom, A. L. eds. Fruit flies (Tephritidae). Phylogeny and evolution of behavior. Boca Raton, CRC Press, p. 3-22.

LOPES, H. S. 1937. Contribuição ao conhecimento do gênero "Stylogaster" Macquart, 1835 (Dipt.: Conopidae). Arquivos do Instituto de Biologia Vegetal 3:257-293.

MCAlpine, J. F. 1989. Phylogeny of the Muscomorpha. In: MCAlPine, J. F.; Peterson, B. V.; Shewell, G. E.; Teskey, H. J.; Vockeroth, J. R. \& Wood, D. M. eds. Manual of Neartic Diptera. Ottawa, Research Branch Agriculture Canada. vol. 3. 248p.

Papavero, N. 1971. Family Conopidae. In: Papavero, N. org. A Catalogue of the Diptera of the America South of the United States. São Paulo, Departamento de Zoologia, Secretaria de Agricultura, vol. 47. p.1-28.

Pape, T. \& Thompson, F. C. eds. 2010. Systema Dipterorum, Version 1.0. Disponível em: <http://www.diptera.org/>. Acessado em 26.08.2012.

Sмітн, K. G. V. 1966. The larva of Thecophora occidensis, with comments upon the biology of Conopidae. Journal of Zoology 149:263-276.

SмiтH, K. G. V. 1967. The biology and taxonomy of the genus Stylogaster Macquart, 1835 (Diptera: Conopidae, Stylogasterinae) in the Ethiopian and Malagassy regions. Transactions of the Royal Entomological Society of London 119:47-69.

Sмiтн, K. G. V. 1969. Further data on the oviposition by the genus Stylogaster Macquart (Diptera: Conopidae, Stylogasterinae) upon adult calyptrate Diptera associated with ants and animal dung. Proceedings of the Royal Entomological Society of London, Series A, General Entomology 44:35-37.

Smith, K. G. V. \& Peterson, B. V. 1987. Conopidae. In: McAlpine, J. F.; Peterson, B. V.; Shewell, G. E.; Teskey, H. J.; Vockeroth, J. R. \& Wood, D. M. eds. Manual of Nearctic Diptera. Ottawa, Research Branch Agriculture Canada. vol. 2. 657p.

SMith, K. G. V. \& VAN Someren, G. R. C. 1970. The identity of Physocephala bimarginipennis Karsch (Diptera, Conopidae) with notes on the immature stages and biology. Journal of Natural History 4:439-446. 